\title{
Stagnation-Point Flow of Casson Fluid in Darcy-Forchheimer Porous Media with Newtonian Heating Effect
}

\author{
${ }^{1}$ Kartini Ahmad and ${ }^{2}$ Zahir Hanouf \\ ${ }^{1}$ Department of Science in Engineering, \\ ${ }^{2}$ Department of Mechanical Engineering, Kulliyyah of Engineering, \\ International Islamic University Malaysia, 50728 Gombak, Kuala Lumpur, Malaysia
}

\begin{abstract}
Flow of Casson fluid in Darcy-Forchheimer porous medium near a stagnation point over a stretching sheet with Newtonian heating effect is investigated in this study. The boundary-layer equations are solved numerically for $\mathrm{f}^{\prime}(0)$ and $\theta(0)$ using the Keller-box method for some values of Casson fluid parameter $(\beta)$, porous media parameter $\left(\kappa_{1}\right)$, inertia-coefficient parameter $\left(\kappa_{2}\right)$, Newtonian heating parameter $(\gamma)$, Prandtl number $(\operatorname{Pr})$ and stretching parameter $(\mathrm{c})$.
\end{abstract}

Key words: Newtonian heating, stretching sheet, Darcy-Forchheimer, medium, boundary-layer, parameter

\section{INTRODUCTION}

The flow behaviour near stagnation-point environs has many applications, particularly in engineering and industry fields. The needs of such study attracted researchers to explore such a situation incorporating various cases, i.e., Newtonian or non-Newtonian fluid, steady or unsteady, two or three-dimensional, etc. Furthermore, the effects of stretching and heat transfer are common aspects in determining the final product. Chiam (1994) discussed stagnation-point flow of inviscid fluid over a linear stretching wall and Mahapatra and Gupta (2002) examined the heat transfer rate in stagnation-point flows towards a stretching sheet. Stagnation-point flow of a micropolar fluid towards a stretching sheet was discussed by Nazar et al. (2004) which was later extended by Attia (2006) taking into account heat generation/absorption and Ishak and Nazar (2009) in the case of permeable sheet. Ahmad et al. (2011) investigated boundary layer flows and heat transfer of micropolar fluids near stagnation point on a stretching vertical surface with prescribed skin friction. Mustafa et al. (2012) solved stagnation-point flow and heat transfer of Casson fluid towards a stretching sheet along with viscous dissipation effects using the HAM method. Shateyi and Marewo (2014) investigated the hydro magnetic boundary layer flow, heat and mass transfer of Casson fluid near a stagnation point over a stretching sheet in the presence of thermal radiation, viscous dissipation and chemical reaction and recently, Medikare et al. (2016) analyzed the effects of viscous dissipation on MHD stagnation-point flow of Casson fluid over a nonlinearly stretching sheet, to name a few. All the above mentioned studies were dedicated to prescribed surface temperature.

According to Merkin (1994), there are four common heating processes, i.e., constant or prescribed wall temperature, constant or prescribed surface heat flux, conjugate condition and Newtonian heating or conjugate convective flow. In modelling convective boundary layer flow, much attention was focused on and only recently has Newtonian heating condition gained considerable attention. Some of the related works can be found in Lesnic et al. (2004), Salleh et al. (2009), Mohamed et al. (2012) and Ahmad et al. (2017).

The study of convective heat transfer in porous media plays an important role in many applications such as thermal insulation, extraction of crude oil, chemical reactors, etc. Many studies have been done on Darcy porous medium which is valid only for slow flows with low permeability. At higher flow rates, there is a departure from linear law and inertial effects. The Darcy-Forchheimer model describes the effect of inertia and viscous forces in porous media (Rawat et al., 2007). Pal and Chatterjee (2012) analyzed two-dimensional stagnation-point flow of an electrically-conducting micropolar fluid over a stretching sheet embedded in non-Darcian porous medium with uniform magnetic and electric fields. Arifin et al. (2016) solved forced convection boundary layer stagnation point slip flow in Darcy-Forchheimer porous medium over a shrinking sheet by shooting method and recently,

Corresponding Author: Kartini Ahmad, Department of Science in Engineering, Kulliyyah of Engineering, International Islamic University Malaysia, 50728 Gombak, Kuala Lumpur, Malaysia 
Mishra et al. (2017) investigated boundary layer magnetohydrodynamic stagnation-point flow past a stretching sheet through non-Darcy porous media in the presence of a heat source/sink. Meanwhile, studies of Casson fluid flow in non-Darcian porous medium were discussed by Prasad et al. (2013), Kumar et al. (2016) and Vijaya et al. (2017). However, to the best knowledge of the researchers, no attempts have been made for stagnation-point Casson fluid flow in non-Darcian porous medium. Hence, motivated by the above mentioned works, this study is aimed at considering Casson fluid flow near a stagnation point in Darcy-Forchheimer porous medium with Newtonian heating effects.

Problem formulation: Consider steady two-dimensional Casson fluid flow near a stagnation point over a stretching sheet immersed in a porous medium. The sheet is stretched with velocity $\mathrm{u}_{\mathrm{w}}(\mathrm{x})=\mathrm{ax}$ and the external velocity of the fluid flow is $\mathrm{u}_{\mathrm{e}}(\mathrm{x})=\mathrm{bx}$ where $\mathrm{a}$ and $\mathrm{b}$ are constants. It is assumed that the sheet is heated due to Newtonian heating and the ambient fluid temperature is $\mathrm{T}_{\infty}$. Under the above conditions, the boundary layer equations that govern the fluid flow are:

$$
\begin{gathered}
\frac{\partial \mathrm{u}}{\partial \mathrm{x}}+\frac{\partial \mathrm{v}}{\partial \mathrm{y}}=0 \\
\mathrm{u} \frac{\partial \mathrm{u}}{\partial \mathrm{x}}+\mathrm{v} \frac{\partial \mathrm{u}}{\partial \mathrm{y}}=\mathrm{u}_{\mathrm{e}} \frac{\mathrm{du} \mathrm{e}_{\mathrm{e}}}{\mathrm{dx}}+\mathrm{v}\left(1+\frac{1}{\beta}\right) \frac{\partial^{2} \mathrm{u}}{\partial \mathrm{y}^{2}}-\frac{v \varepsilon}{\kappa}\left(\mathrm{u}-\mathrm{u}_{\mathrm{e}}\right)-\frac{\mathrm{F} \varepsilon^{2}}{\sqrt{\kappa}}\left(\mathrm{u}^{2}-\mathrm{u}_{\mathrm{e}}^{2}\right) \\
\mathrm{u} \frac{\partial \mathrm{T}}{\partial \mathrm{x}}+\mathrm{v} \frac{\partial \mathrm{T}}{\partial \mathrm{y}}=\alpha \frac{\partial^{2} \mathrm{~T}}{\partial \mathrm{y}^{2}}
\end{gathered}
$$

Where:

$\mathrm{u}$ and $\mathrm{v}=$ The velocity components along the $\mathrm{x}$ and $\mathrm{y}$ directions, respectively

$\mathrm{v} \quad=$ The kinematic viscosity

$\beta=$ The non-Newtonian (Casson) fluid parameter

$\varepsilon \quad=$ The dimensionless stretching parameter

$\kappa=$ The permeability of the porous medium

$\mathrm{T}=$ The Temperature

$\alpha \quad=$ The thermal diffusivity

It should be pointed out that the last two terms in Eq. 2 represents the Darcy-Forchheimer porous medium. The appropriate boundary conditions for the problem are:

$$
\begin{aligned}
& \mathrm{u}=\mathrm{u}_{\mathrm{w}}, \quad \mathrm{v}=0, \quad \frac{\partial \mathrm{T}}{\partial \mathrm{y}}=-\mathrm{h}_{\mathrm{f}} \mathrm{T}(\mathrm{NH}) \quad \text { at } \quad \mathrm{y}=0 \\
& \mathrm{u} \rightarrow \mathrm{u}_{\mathrm{e}}, \quad \mathrm{T} \rightarrow \mathrm{T}_{\infty} \quad \text { as } \quad \mathrm{y} \rightarrow \infty
\end{aligned}
$$

with $\mathrm{h}$ being the heat transfer coefficient. Equation 1-3 subject to Eq. 4 can be transformed to ordinary differential equations by the following similarity transformation (Mohamed et al., 2013):

$$
\Psi=\sqrt{u_{e} v x} f(\eta), \quad \eta=\sqrt{\frac{u_{e}}{v x}} y, \quad \theta(\eta)=\frac{T-T_{\infty}}{T_{\infty}}(N H)
$$

Where:

$\Psi=$ The stream function defined in usual fashion, i.e., $\mathrm{u}$ $=\partial \Psi / \partial \mathrm{y}$ and $\mathrm{v}=\partial \Psi / \partial \mathrm{x}$

$\eta=$ The similarity variable

$f(\eta)$ and $\theta(\eta)$ are the dimensionless stream function and dimensionless temperature, respectively and prime denotes differentiation with respect to $\eta$. Making use of Eq. 5 into Eq. 1-3, Eq. 1 is automatically satisfied and Eq. 2-3 reduced to:

$$
\begin{gathered}
\left(1+\frac{1}{\beta}\right) \mathrm{f}^{\prime \prime \prime}+\mathrm{ff}^{\prime \prime}-\mathrm{f}^{\prime 2}-\kappa_{1}\left(\mathrm{f}^{\prime}-1\right)-\kappa_{2}\left(\mathrm{f}^{\prime 2}-1\right)+1=0 \\
\theta^{\prime \prime}+\operatorname{Prf} \theta^{\prime}=0
\end{gathered}
$$

Similarly, the boundary conditions Eq. 4 after implementing Eq. 5 is reduced to:

$$
\begin{aligned}
& f(\eta)=0, \quad f^{\prime}(\eta)=c, \quad \theta^{\prime}(\eta)=-\gamma[1+\theta(0)] \text { at } \eta=0 \\
& f^{\prime}(\eta) \rightarrow 1, \quad \theta(\eta) \rightarrow 0 \quad \text { as } \eta \rightarrow \infty,
\end{aligned}
$$

where, prime denotes differentiation with respect to $\eta$, $\kappa_{1}=v \varepsilon / \kappa b$ is the porous media parameter, $\kappa_{2}=\mathrm{F}^{2} \mathrm{X} / \sqrt{\kappa}$ is the inertia-coefficient parameter, $\operatorname{Pr}=\mu c_{p} / k$ is the Prandtl number, $\mathrm{c}=\mathrm{a} / \mathrm{b}(\geq 0)$ is the stretching parameter and $\gamma=h_{\mathrm{f}} \sqrt{\mathrm{v} / \mathrm{b}}$ is the conjugate parameter for the Newtonian heating.

\section{RESULTS AND DISCUSSION}

Equation 6 and 7 subjected to Eq. 8 were solved using the method described by Cebeci and Bradshaw (1988) for arbitrary values of Casson fluid parameter $(\beta)$, porous media parameter $\left(\kappa_{1}\right)$, inertia-coefficient parameter $\left(\kappa_{2}\right)$, Newtonian heating parameter $(\gamma)$, stretching parameter (c) and Prandtl number ( $\mathrm{Pr})$. As far as engineering interest is concerned, there are two physical quantities of interest, i.e., the skin friction coefficient and the local Nusselt number which can be found once the values of $f^{\prime \prime}(0)$ and $\theta^{\prime}(0)$ are secured. Retained that the value of $\theta^{\prime}(0)$ can be acquired once $\theta(0)$ is known as given in Eq. 8 . 


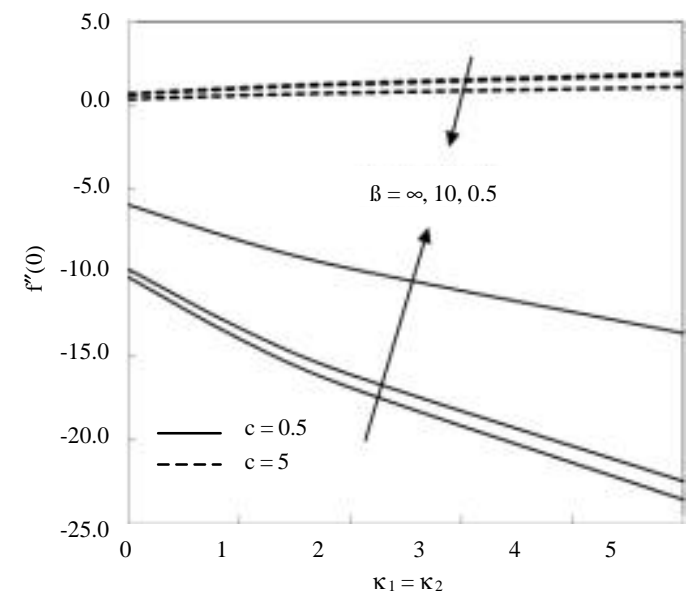

Fig. 1: Variations of $f$ " $(0)$ for various values of $\kappa_{1}, \kappa_{2}$ and $\beta$ when $\mathrm{c}=0.5$ and 5 , respectively

Table 1: Values of $f^{\prime \prime}(0)$ when $\kappa_{1}=\kappa_{2}=0$ and $\beta=8$

\begin{tabular}{llll}
\hline c & Present results & Mohamed et al. (2013) & Wang (2008) \\
0 & 1.2315306 & 1.2325877 & 1.232588 \\
0.5 & 0.7121119 & 0.7132949 & 0.71330 \\
1 & 0 & 0 & 0 \\
2 & -1.8859019 & -1.8873066 & -1.88731 \\
\hline
\end{tabular}

Table 2: Values of $f^{\prime}(0)$ for various values $\beta, \kappa_{1}, \kappa_{2}$ and $c$

\begin{tabular}{lll}
\hline$\kappa 1=\kappa 2 / \mathrm{c}$ & $\beta=0.5$ & $\beta=10$ \\
\hline $\mathbf{0}$ & 0.7126263 & 1.1741682 \\
0 & 0.4106638 & 0.6789219 \\
0.5 & 0 & 0 \\
1 & -1.0882539 & -1.7980719 \\
2 & & \\
$\mathbf{0 . 1}$ & 0.7630589 & 1.2607018 \\
0 & 0.4365975 & 0.7217265 \\
0.5 & 0 & 0 \\
1 & -1.1420484 & -1.8868910 \\
2 & & \\
$\mathbf{0 . 5}$ & 0.9444179 & 1.5602419 \\
0 & 0.5281054 & 0.8727675 \\
0.5 & 0 & 0 \\
1 & -1.3368236 & -2.2084359 \\
2 & & \\
\hline
\end{tabular}

Values obtained for $\mathrm{f}^{\prime \prime}(0)$ when $\kappa_{1}=\kappa_{2}=0$ and $\beta=\infty$ in our study are compared with Wang (2008) and Mohamed et al. (2013) as tabulated in Table 1 and were found to be satisfactory. It should be pointed out that Eq. 6 and 7 are decoupled. As such, $f^{\prime \prime}(0)$ is independent of Pr number and $\gamma$. The effects of $\beta, \kappa_{1}$ and $\kappa_{2}$ on $f^{\prime \prime}(0)$ are given in Table 2 for various values of $c$. It is seen that at fixed $\kappa_{1}$ and $\kappa_{2}$ and as $\beta$ increases, $f^{\prime \prime}(0)$ increases for $c<1$ and contrary impact is seen for $c>1$. These results are further supported by the graph depicted in Fig. 1. Increments of $\kappa_{1}$ and $\kappa_{2}$ are found to increase and decrease $\mathrm{f}^{\prime \prime}(0)$ for $\mathrm{c}<1$ and $\mathrm{c}>1$, respectively. However, $\mathrm{c}=1$ impacted to nil $\mathrm{f}^{\prime \prime}(0)$ throughout the boundary layer for any values of $\kappa_{1}, \kappa_{2}$ and $\beta$.

The effect of $c$ does not only influence the $f^{\prime \prime}(0)$ but also the surface temperature $\theta(0)$. The accession of
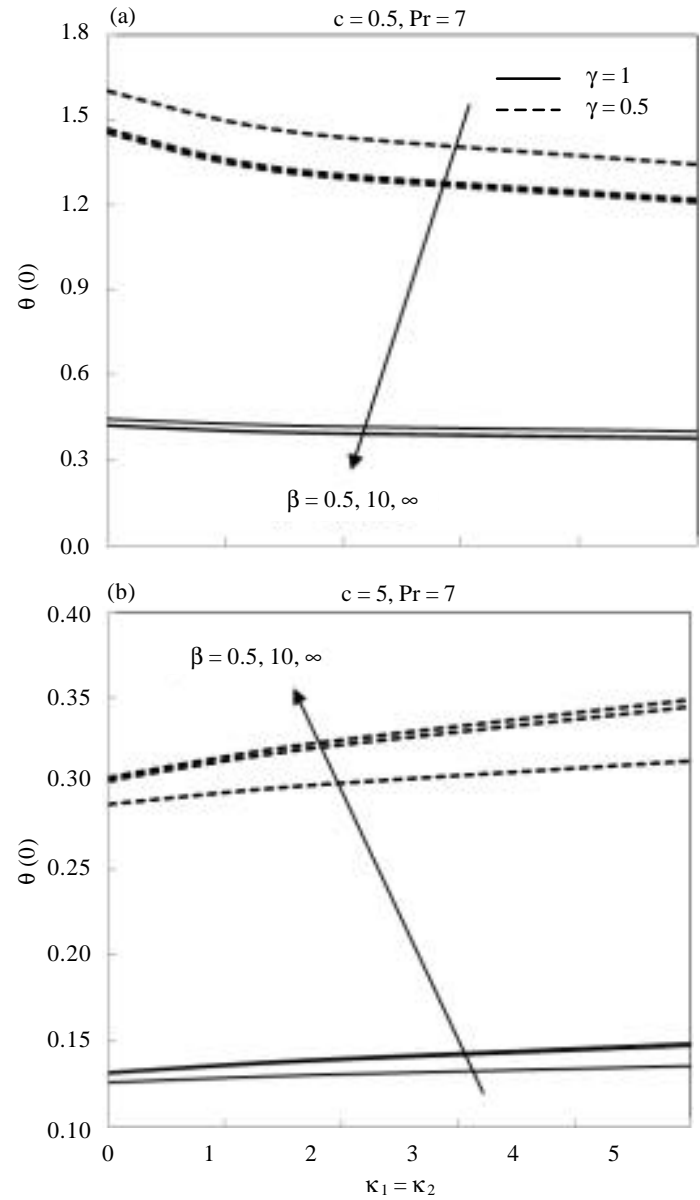

Fig. 2: Variations of $\theta(0)$ for various values of $\kappa_{1}, \kappa_{2}, \beta$ and $\gamma$ when $\operatorname{Pr}=7$ and $c=0.5$ and 5 , respectively

stretching parameter $\mathrm{c}$ results in low surface temperature $\theta(0)$ at fixed $\kappa_{1}, \kappa_{2}$ and $\gamma$. However, as $\kappa_{1}$ and $\kappa_{2}$ increase, $\theta(0)$ also experience the same contrary behavior for $c<1$ and $c>1$ as depicted in Fig. 2, i.e., $\theta(0)$ is found to decrease as $\kappa_{1}$ and $\kappa_{2}$ increase for $\mathrm{c}<1 \quad(\mathrm{c}=0.5)$ and reverse features for $\mathrm{c}>1(\mathrm{c}=5)$. The property behaviour of the fluid itself from Newtonian $(\beta=\infty)$ to non-Newtonian fluid $(\beta=\infty)$ is seen to give higher surface temperature $\theta(0)$ for $\mathrm{c}<1$ and an opposite trend occurs for $\mathrm{c}>1$.

On top of that, the increment of $\kappa_{1}$ and $\kappa_{2}$ had an outstanding impact on $\theta(0)$ for $\gamma=1$ as compared to $\gamma=0.5$, i.e., the impact of $\kappa_{1}$ and $\kappa_{2}$ are not very critical. As the sheet was being heated due to Newtonian heating $\gamma$, the surface temperature $\theta(0)$ for $\gamma=1$ was higher than $\gamma=0.5$ which was expected to happen.

Pr number is very much significant in determining the heat transfer rate at the surface. The effect of this parameter can be seen in Fig. 3. It is very clear that increments of the Pr number results in the decrement of surface temperature $\theta(0)$. This is because as $\operatorname{Pr}$ increases, 

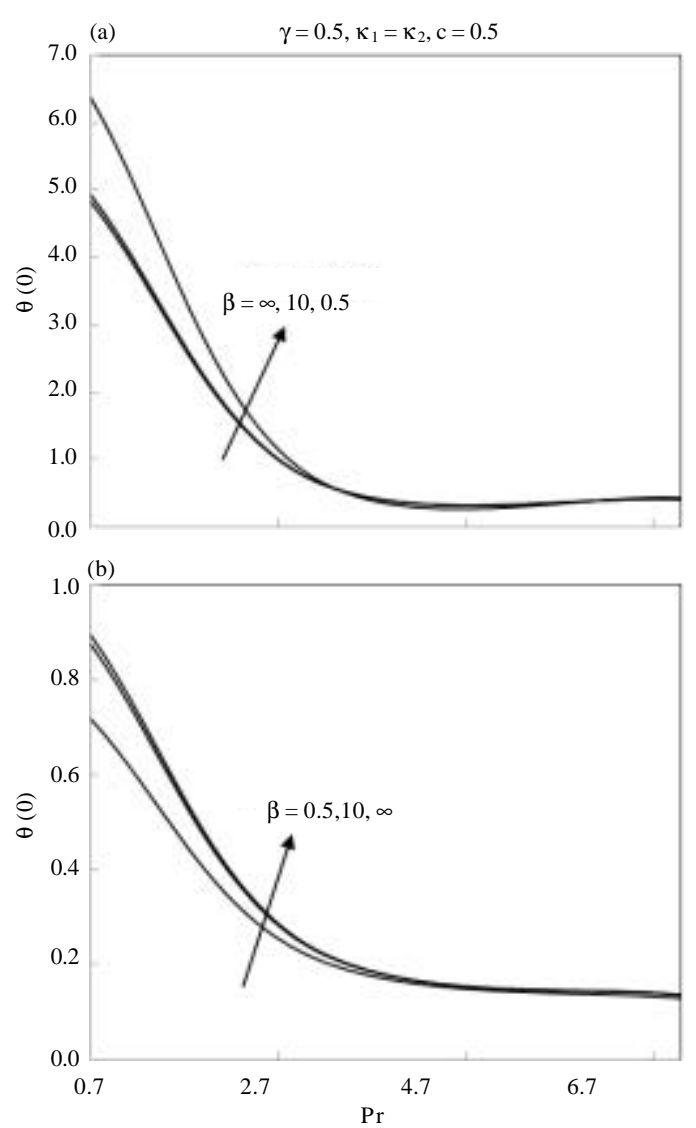

Fig. 3: Variations of $\theta(0)$ for various values of $\beta$ and $\operatorname{Pr}$ when $\gamma=0.5, \kappa_{1}=\kappa_{2}=0.5$ and $\mathrm{c}=0.5$ and 5 , respectively

the thermal diffusivity decreases which in turn decreases the thermal energy. Additionally, the effect of stretching parameter $\mathrm{c}$ is found to decrease the surface temperature. Flow of Casson fluid is very much profound for small values of $\operatorname{Pr}$ number $(\operatorname{Pr}<<3)$ where Casson fluid $\beta$ is seen to offer variations in the surface temperature, unlike for $\mathrm{Pr} \gg>3$. It is observed that the non-Newtonian fluid caused higher $\theta(0)$ for $c<1$ and a contrary trend was observed for $c>1$. This scenario was only valid for $\operatorname{Pr}<<3$.

The velocity and temperature profiles for $\beta=\gamma=0.5$ and $\mathrm{Pr}=0.7$ are depicted in Fig. 4. The velocity profile is seen to have inverted boundary layer structured when c $>1$ and no momentum boundary layer was formed when $\mathrm{c}=1$. This is in compliance with the results obtained in Table 1 and 2. As $\kappa_{1}$ and $\kappa_{2}$ increase, the velocity decreases within the boundary layer for $c>1$ which results in the decrement of $\mathrm{f}^{\prime \prime}(0)$ as demonstrated in Fig. 1. It should be noted that the opposite situation occurs for $c<1$. Nevertheless, the temperature distribution within the boundary layer can also be seen on the right of Fig. 4 .
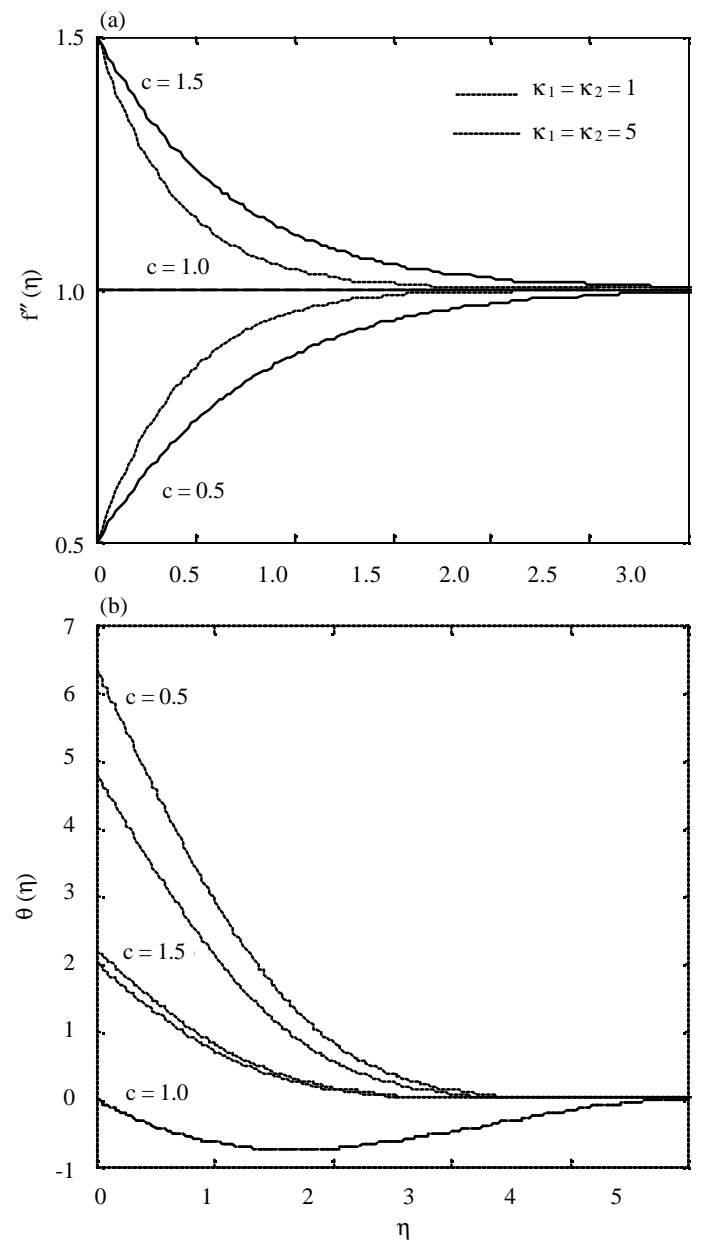

Fig. 4: Velocity and temperature profiles for $\beta=\gamma=0.5$ and $\operatorname{Pr}=0.7$

\section{CONCLUSION}

In this study, we discussed the effect of stretching sheet and Newtonian heating for flow of Casson fluid embedded in Darcy-Forchheimer porous medium near a stagnation point. It can be concluded that $\mathrm{c}$ plays a major role in determining $\mathrm{f}^{\prime \prime}(0)$ which later affects surface temperature $\theta(0)$. The repercussion of the stretching parameter c on $\mathrm{f}^{\prime \prime}(0)$ and $\theta(0)$ for various values of Casson fluid parameter $\beta$, porous media parameter $\kappa_{1}$, inertiacoefficient parameter $\kappa_{2}$, Prandtl number Pr and Newtonian heating parameter $\gamma$ very much depends on whether $c>1$ or $\mathrm{c}<1$.

\section{ACKNOWLEDGEMENT}

This research is supported by FRGS grant (FRGS1 5-228-0469) from the Ministry of Higher Education Malaysia. 


\section{REFERENCES}

Ahmad, K., R. Nazar and I. Pop, 2011. Boundary layer flows and heat transfer of micropolar fluids near the stagnation point on a stretching vertical surface with prescribed skin friction. Intl. J. Miner. Metall. Mater., 18: $502-507$.

Ahmad, K., Z. Hanouf and A. Ishak, 2017. MHD Casson nanofluid flow past a wedge with Newtonian heating. Eur. Phys. J. Plus, Vol. 132,

Arifin, N., S. Abu Bakar, R. Nazar, F. and I. Pop, 2016. Forced convection boundary layer stagnation-point flow in darcy-forchheimer porous medium past a shrinking sheet. Front. Heat Mass Transfer FHMT., 7: 1-6.

Attia, H.A., 2006. Heat transfer in a stagnation point flow of a micropolar fluid over a stretching surface with heat generation/absorptio n. Danjiang. J. Sci. Technol., 9: 299-305.

Cebeci, T. and P. Bradshaw, 1988. Physical and Computational Aspects of Convective Heat Transfer. 2nd Edn., Springer, New York, USA., ISBN:9783540968214, Pages: 487.

Chiam, T.C., 1994. Stagnation-point flow towards a stretching plate. J. Phys. Soc. Japan, 63: 2443-2444.

Ishak, A. and R. Nazar, 2009. Stagnation point flow over a stretching permeable sheet micropolar fluid. J. Qual. Meas. Anal. JQMA., 5: 45-50.

Kumar, P.S., M.B. Devi and K. Gangadhar, 2016. Slip velocity on a casson fluid flow over a vertical porous surface with heat generation or absorption in a non-darcy porous medium. Intl. J. Appl. Math. Sci., 9: 77-101.

Lesnic, D., D.B. Ingham, I. Pop and C. Storr, 2004. Free convection boundary-layer flow above a nearly horizontal surface in a porous medium with Newtonian heating. Heat Mass Transfer, 40: 665-672.

Mahapatra, T.R. and A.S. Gupta, 2002. Heat transfer in stagnation-point flow towards a stretching sheet. Heat Mass Transfer, 38: 517-521.

Medikare, M., S. Joga and K.K. Chidem, 2016. MHD stagnation point flow of a casson fluid over a nonlinearly stretching sheet with viscous dissipation. Am. J. Comput. Math., 6: 37-48.

Merkin, J.H., 1994. Natural-convection boundary-layer flow on a vertical surface with Newtonian heating. Int. J. Heat Fluid Flow, 15: 392-398.
Mishra, S.R., B. Nayak and R.P. Sharma, 2017. MHD stagnation-point flow past over a stretching sheet in the presence of non-Darcy porous medium and heat source/sink. Defect Diffusion Forum, 374: 92-105.

Mohamed, M.K.A., M.Z. Salleh, R. Nazar and A. Ishak, 2012. Stagnation point flow over a stretching sheet with Newtonian heating. Sains Malays, 41: 1467-1473.

Mohamed, M.K.A., M.Z. Salleh, R. Nazar and A. Ishak, 2013. Numerical investigation of stagnation point flow over a stretching sheet with convective boundary conditions. Boundary Value Prob., 2013: $1-10$.

Mustafa, M., T. Hayat, P. Ioan and A. Hendi, 2012. Stagnation-point flow and heat transfer of a Casson fluid towards a stretching sheet. Zeitschrift für Naturforsch. A., 67: 70-76.

Nazar, R., N. Amin, D. Filip and I. Pop, 2004. Stagnation point flow of a micropolar fluid towards a stretching sheet. Intl. J. Non Linear Mech., 39: 1227-1235.

Pal, D. and S. Chatterjee, 2012. MHD Non-Darcy mixed convection stagnation-point flow of a micropolar fluid towards a stretching sheet with radiation. Chem. Eng. Commun., 199: 1169-1193.

Prasad, V.R., A.S. Rao and O.A. Beg, 2013. Flow and heat transfer of casson fluid from a horizontal circular cylinder with partial slip in non-darcy porous medium. J. Appl. Comput. Math., Vol. 2. 10.4172/2168-9679.1000127

Rawat, S., R. Bhargava and A.O. Beg, 2007. A finite element study of transport phenomena in MHD micropolar flow in a Darcy-Forchheimer porous medium. Proceedings of the World Congress on Engineering and Computer Science WCECS 2007 , October 24-26, 2007, WCECS, San Francisco, USA., ISBN:978-988-98671-6-4, pp: 169-174.

Salleh, M.Z., R. Nazar and I. Pop, 2009. Forced convection boundary layer flow at a forward stagnation point with Newtonian heating. Chem. Eng. Commun., 196: 987-996.

Shateyi, S. and G.T. Marewo, 2014. Numerical analysis of $\mathrm{MHD}$ stagnation point flow of Casson fluid, heat and mass transfer over a stretching sheet. Adv. Appl. Pure Math., 2014: 128-132.

Vijaya, N., K. Sreelakshmi and G. Sarojamma, 2017. Nonlinear radiation effect on casson fluid saturated non-darcy porous medium. Intl. J. Math. Arch., 8: 39-52.

Wang, C.Y., 2008. Stagnation flow towards a shrinking sheet. Int. J. Non-Linear Mech., 43: 377-382. 